arXiv:1309.4939

\title{
A light Higgs scalar in the NMSSM confronted with the latest LHC Higgs data
}

\author{
Junjie $\mathrm{Cao}^{1,2}$, Fangfang Ding ${ }^{1}$, Chengcheng $\mathrm{Han}^{3}$, Jin Min Yang ${ }^{3}$, Jingya Zhu ${ }^{3}$ \\ 1 Department of Physics, Henan Normal University, Xinxiang 453007, China \\ 2 Center for High Energy Physics, Peking University, Beijing 100871, China \\ 3 State Key Laboratory of Theoretical Physics, \\ Institute of Theoretical Physics, Academia Sinica, Beijing 100190, China
}

\begin{abstract}
In the Next-to-Minimal Supersymemtric Standard Model (NMSSM), one of the neutral Higgs scalars (CP-even or CP-odd) may be lighter than half of the SM-like Higgs boson. In this case, the SM-like Higgs boson $h$ can decay into such a light scalar pair and consequently the $\gamma \gamma$ and $Z Z^{*}$ signal rates at the LHC will be suppressed. In this work, we examine the constraints of the latest LHC Higgs data on such a possibility. We perform a comprehensive scan over the parameter space of the NMSSM by considering various experimental constraints and find that the LHC Higgs data can readily constrain the parameter space and the properties of the light scalar, e.g., at $3 \sigma$ level this light scalar should be highly singlet dominant and the branching ratio of the SM-like Higgs boson decay into the scalar pair should be less than about 30\%. Also we investigate the detection of this scalar at various colliders. Through a detailed Monte Carlo simulation we find that under the constraints of the current Higgs data this light scalar can be accessible at the LHC-14 with an integrated luminosity over $300 \mathrm{fb}^{-1}$.

PACS numbers: 14.80.Da, 12.60.Jy
\end{abstract}




\section{INTRODUCTION}

The existence of a new scalar has been discovered by the ATLAS and CMS collaborations with a significance of $9 \sigma$ and $7 \sigma$, respectively [1 4]. So far the mass of this scalar is rather precisely determined to be around $125 \mathrm{GeV}$, and its other properties, albeit with large experimental uncertainties, agree with the Standard Model (SM) prediction [4, 5]. In spite of this, this newly discovered scalar has been interpreted in various new physic models since the SM has the gauge hierarchy problem and cannot provide a dark matter candidate. The studies in this direction have been carried out intensively in low energy supersymmetric models and the NMSSM was found to be most favorved [6-13].

In this work we focus on the NMSSM, which is the simplest extension of the MSSM with one extra gauge singlet Higgs field [14]. One virtue of such an extension is that it provides a dynamical mechanism for the generation of the parameter $\mu$ and thus solves the so-called $\mu$-problem suffered by the MSSM [15]. Another virtue is that the interactions of the singlet field in the Higgs sector give a new contribution to the tree-level mass of the SM-like Higgs boson and thus alleviate the little hierarchy problem [10, 16]. For the LHC phenomenology, one notable feature of the NMSSM is that a Higgs scalar (CP-even or CP-odd) may be rather light [17, 18], which can affect the signals of the sparticles at the LHC [19, 20]. For example, if the lightest supersymmetric particle is singlino-like, squarks may decay dominantly as [20] $\tilde{q} \rightarrow q \tilde{\chi}_{2,3}^{0} \rightarrow q \tilde{\chi}_{1}^{0} S \rightarrow q \tilde{\chi}_{1}^{0} b \bar{b}$, where $\tilde{\chi}_{2}^{0}$ and $\tilde{\chi}_{3}^{0}$ represent the second and the third lightest neutralino respectively, and $S$ denotes a light scalar.

We note that, if this scalar is lighter than half of the SM-like Higgs boson, the SM-like Higgs boson can decay exotically into the light scalar pair [21 23]. Since the width of the Higgs boson in the SM is quite narrow (about $4 \mathrm{MeV}$ ), such an exotic decay may have a sizable branching ratio. This in return can suppress greatly the visible signals of the SM-like Higgs boson at the LHC. Motivated by this observation, we in this work investigate the constraints of the latest LHC Higgs data on the properties of such a light scalar. We will also study the detection of this scalar at the LHC-14 via a detailed Monte Carlo simulation.

The outline of this paper is as follows. In Section II we briefly review the NMSSM model. Then in Section III we scan the parameter space of the NMSSM under current experimental constraints. In Section IV the properties of the light scalar are analyzed and its detection at the LHC-14 is studied via a detailed Monte Carlo simulation. Finally, we present our 
conclusion in Section V.

\section{THE HIGGS SECTOR OF THE NMSSM}

As one of the most economical extensions of the MSSM, the NMSSM contains two SU(2) doublet Higgs fields and one gauge singlet Higgs field [14]. Traditionally, these fields are labeled by

$$
\hat{H}_{u}=\left(\begin{array}{c}
H_{\mathrm{u}}^{+} \\
v_{u}+\frac{\phi_{u}+i \varphi_{u}}{\sqrt{2}}
\end{array}\right), \hat{H}_{d}=\left(\begin{array}{c}
v_{d}+\frac{\phi_{d}+i \varphi_{d}}{\sqrt{2}} \\
H_{\mathrm{d}}^{-}
\end{array}\right), \hat{S}=v_{s}+\frac{\phi_{s}+i \varphi_{s}}{\sqrt{2}},
$$

where $H_{i}^{+}, \phi_{i}$ and $\varphi_{i}(i=u, d)$ represent the charged, neutral CP-even and neutral CP-odd component fields respectively, and $v_{u}, v_{d}$ and $v_{s}$ are the vacuum expectation values with $v_{u} / v_{d}=\tan \beta$ and $\sqrt{v_{u}^{2}+v_{d}^{2}}=v \equiv 174 \mathrm{GeV}$. Since one purpose of the extension is to solve the $\mu$-problem of the MSSM, a $Z_{3}$ symmetry is implemented in the construction of the superpotential to avoid the appearance of parameters with mass dimension. Consequently, the superpotential and the soft breaking terms in the NMSSM are given by [14]

$$
\begin{aligned}
W^{\mathrm{NMSSM}} & =W_{F}+\lambda \hat{H}_{u} \cdot \hat{H}_{d} \hat{S}+\frac{1}{3} \kappa \hat{S^{3}}, \\
V_{\mathrm{soft}}^{\mathrm{NMSSM}} & =\tilde{m}_{u}^{2}\left|H_{u}\right|^{2}+\tilde{m}_{d}^{2}\left|H_{d}\right|^{2}+\tilde{m}_{S}^{2}|S|^{2}+\left(\lambda A_{\lambda} S H_{u} \cdot H_{d}+\frac{1}{3} \kappa A_{\kappa} S^{3}+\text { h.c. }\right),
\end{aligned}
$$

where $\hat{H}_{u}, \hat{H}_{d}$ and $\hat{S}$ are Higgs superfields, $W_{F}$ is the superpotential of the MSSM without the $\mu$-term, and $\tilde{m}_{u}, \tilde{m}_{d}, \tilde{m}_{S}, A_{\lambda}$ and $A_{\kappa}$ are soft-breaking parameters.

In order to present the mass matrices of the Higgs fields in a physical way, we redefine the Higgs fields as [24]

$$
H_{1}=\cos \beta H_{u}-\varepsilon \sin \beta H_{d}^{*}, \quad H_{2}=\sin \beta H_{u}+\varepsilon \cos \beta H_{d}^{*}, \quad H_{3}=S,
$$

where $\varepsilon_{12}=\varepsilon_{21}=-1$ and $\varepsilon_{11}=\varepsilon_{22}=0$. With such a definition, $H_{i}(i=1,2,3)$ are given by

$$
H_{1}=\left(\begin{array}{c}
H^{+} \\
\frac{S_{1}+i P_{1}}{\sqrt{2}}
\end{array}\right), \quad H_{2}=\left(\begin{array}{c}
G^{+} \\
v+\frac{S_{2}+i G^{0}}{\sqrt{2}}
\end{array}\right), \quad H_{3}=v_{s}+\frac{1}{\sqrt{2}}\left(S_{3}+i P_{2}\right),
$$

where $\phi_{s}$ and $\varphi_{s}$ in Eq.(1) are rewritten as $S_{3}$ and $P_{2}$ respectively. Obviously, the field $H_{2}$ corresponds to the SM Higgs field with $G^{+}$and $G^{0}$ denoting Goldstone bosons, and $S_{2}$ representing the SM Higgs boson. 
In the CP-conserving NMSSM, the fields $S_{1}, S_{2}$ and $S_{3}$ mix to form three (instead of two in the MSSM) physical CP-even Higgs bosons $h_{i}(i=1,2,3)$. In the basis $\left(S_{1}, S_{2}, S_{3}\right)$, the elements of the corresponding mass matrix are given by [24]

$$
\begin{aligned}
& M_{11}^{2}=M_{A}^{2}+\left(m_{Z}^{2}-\lambda^{2} v^{2}\right) \sin ^{2} 2 \beta, \\
& M_{12}^{2}=-\frac{1}{2}\left(m_{Z}^{2}-\lambda^{2} v^{2}\right) \sin 4 \beta, \\
& M_{13}^{2}=-\left(\frac{M_{A}^{2}}{2 \mu / \sin 2 \beta}+\kappa v_{s}\right) \lambda v \cos 2 \beta, \\
& M_{22}^{2}=m_{Z}^{2} \cos ^{2} 2 \beta+\lambda^{2} v^{2} \sin ^{2} 2 \beta, \\
& M_{23}^{2}=2 \lambda \mu v\left[1-\left(\frac{M_{A}}{2 \mu / \sin 2 \beta}\right)^{2}-\frac{\kappa}{2 \lambda} \sin 2 \beta\right], \\
& M_{33}^{2}=\frac{1}{4} \lambda^{2} v^{2}\left(\frac{M_{A}}{\mu / \sin 2 \beta}\right)^{2}+\kappa v_{s} A_{\kappa}+4\left(\kappa v_{s}\right)^{2}-\frac{1}{2} \lambda \kappa v^{2} \sin 2 \beta .
\end{aligned}
$$

Similarly, the fields $P_{1}$ and $P_{2}$ mix to form two physical CP-odd Higgs bosons $A_{i}(i=1,2)$, and in the basis $\left(P_{1}, P_{2}\right)$ the mass matrix elements for CP-odd Higgs sector are given by

$$
\begin{aligned}
& M_{-11}^{2}=M_{A}^{2}=\frac{2 \mu}{\sin 2 \beta}\left(A_{\lambda}+\kappa v_{s}\right), \\
& M_{-22}^{2}=M_{P}^{2}=\lambda^{2} v^{2}\left(\frac{M_{A}}{2 \mu / \sin 2 \beta}\right)^{2}+\frac{3}{2} \lambda \kappa v^{2} \sin 2 \beta-3 \kappa v_{s} A_{\kappa}, \\
& M_{-12}^{2}=\lambda v \frac{M_{A}^{2}}{2 \mu / \sin 2 \beta}-3 \lambda \kappa v_{s} v .
\end{aligned}
$$

About the Higgs sector of the NMSSM, the following points should be noted:

- Compared with the MSSM where only two parameters are involved in the Higgs sector, six parameters are needed to describe the Higgs sector of the NMSSM [14]. These parameters are usually chosen as

$$
\lambda, \quad \kappa, \quad \tan \beta=\frac{v_{u}}{v_{d}}, \quad \mu=\lambda v_{s}, \quad M_{A}^{2}=\frac{2 \mu}{\sin 2 \beta}\left(A_{\lambda}+\kappa v_{s}\right), \quad M_{P} .
$$

Since the NMSSM predicts one more CP-odd Higgs field than the MSSM, $M_{A}$ here no longer represents the mass of one CP-odd state. Obviously, the Higgs sector of the NMSSM is quite complicated.

- After diagonalizing the mass matrix in Eq.(6) , one can get the mass eigenstates of CP-even states $h_{i}$ as

$$
h_{i}=\sum_{j=1}^{3} V_{i j} S_{j}
$$


where $V_{i j}$ is the element of the transition matrix satisfying $V_{i 1}^{2}+V_{i 2}^{2}+V_{i 3}^{2}=1$, and it represents the component of $S_{j}$ in the physical state $h_{i}$. In the following, we assume $m_{h_{3}}>m_{h_{2}}>m_{h_{1}}$, and call the state whose squared component coefficient of $S_{2}$ larger than 0.5 the SM-like Higgs boson.

Similarly, the mass eigenstates of the $\mathrm{CP}$-odd states $A_{i}$ are given by

$$
A_{i}=\sum_{j=1}^{2} U_{i j} P_{j}
$$

If the lighter state $A_{1}$ satisfies $U_{11}^{2}>0.5$, we call it doublet dominated; otherwise we call it singlet dominated.

- Like the MSSM, the mass of the SM-like Higgs boson may be greatly changed by the radiative corrections. Denoting the loop-corrected mass matrix of the CP-even states by $\tilde{M}^{2}$, one can conclude that for $\tilde{M}_{11}^{2}>\tilde{M}_{33}^{2}>\tilde{M}_{22}^{2}$, the state $h_{1}$ corresponds to the SM-like Higgs boson, while for $\tilde{M}_{11}^{2}>\tilde{M}_{22}^{2}>\tilde{M}_{33}^{2}$, the state $h_{2}$ is the SM-like Higgs boson [6].

- Obviously, in order to get a light CP-odd Higgs boson, either $M_{A}$ or $M_{P}$ should be moderately small, and a large $M_{-12}^{2}$ can further suppress the mass of the lighter CPodd state.

\section{NUMERICAL RESULT AND DISCUSSION}

In this work, we first perform a comprehensive scan over the parameter space of the NMSSM by considering various experimental constraints. Then for the surviving samples we investigate the features of the light scalar. Since for the NMSSM there are too many free parameters, we make the following assumptions to simplify our analysis:

- First, we note that the first two generation squarks have little effects on the Higgs sector of the NMSSM, and the LHC search for SUSY particles implies that they should be heavier than $1 \mathrm{TeV}$. So we fix all soft breaking parameters (i.e. soft masses and trilinear coefficients) in this sector to be $2 \mathrm{TeV}$. We checked that our conclusions are not sensitive to this sector. 
- Second, considering that the third generation squarks can change significantly the properties of the Higgs bosons, we set free all soft parameters in this sector except that we assume $m_{U_{3}}=m_{D_{3}}$ and $A_{t}=A_{b}$ to reduce the number of free parameters.

- Third, since we require the NMSSM to explain the discrepancy of the measured value of the muon anomalous magnetic moment from its SM prediction, i.e., $a_{\mu}^{e x p}-a_{\mu}^{S M}=$ $(28.7 \pm 8.0) \times 10^{-10}[25]$, we assume all soft breaking parameters in the slepton sector to take a common value $m_{\tilde{l}}$ and treat $m_{\tilde{l}}$ as a free parameter.

- Finally, we note that our results are not sensitive to gluino mass, we fix it at $2 \mathrm{TeV}$. We also assume the grand unification relation $3 M_{1} / 5 \alpha_{1}=M_{2} / \alpha_{2}$ for electroweak gaugino masses.

With above assumptions, we use the package NMSSMTools-4.0.0 [26] to scan randomly the free parameters of the model in the following ranges

$$
\begin{aligned}
& 0.1 \leq \lambda, \kappa \leq 0.8, \quad 1 \mathrm{GeV} \leq M_{A}, M_{P} \leq 2 \mathrm{TeV} \\
& 1 \leq \tan \beta \leq 30, \quad 100 \mathrm{GeV} \leq \mu, M_{2}, m_{\tilde{l}} \leq 1 \mathrm{TeV} \\
& \left|A_{t}\right| \leq 5 \mathrm{TeV}, \quad 100 \mathrm{GeV} \leq M_{Q_{3}}, M_{U_{3}} \leq 2 \mathrm{TeV}
\end{aligned}
$$

In our scan, we only keep the samples that predict a SM-like Higgs boson $h$ with mass around $125 \mathrm{GeV}$ (e.g. $123 \mathrm{GeV} \leq m_{h} \leq 127 \mathrm{GeV}$ ) along with a light neutral Higgs scalar (CP-even or CP-odd) with mass less than $m_{h} / 2$, and meanwhile satisfy the following constraints:

(1) All the constraints implemented in the package NMSSMTools-4.0.0. These constraints are from the vacuum stability, the LEP search for sparticles (including lower bounds on various sparticle masses, the upper bounds on the neutralino pair production rates), the $Z$-boson invisible decay, the $\Upsilon$ decay into a light scalar plus one photon [27], the $B$-physics observables (such as the branching ratios for $B \rightarrow X_{\mathrm{s}} \gamma, B_{s} \rightarrow \mu^{+} \mu^{-}$and $B^{+} \rightarrow \tau^{+} \nu_{\tau}$, and the mass differences $\Delta M_{d}$ and $\Delta M_{s}$ ) [25, 28, 29], the discrepancy of the muon anomalous magnetic moment, the dark matter relic density [30] and the XENON100(2012) limits on the scattering rate of dark matter with nucleon [31, 32]. In imposing the constraint from a certain observable which has an experimental central value, we use its latest measured result and require the NMSSM to explain the result at $2 \sigma$ level. 
(2) The constraints from the search for Higgs bosons at the LEP, the Tevatron and the LHC. We implement these constraints by the package HiggsBounds-4.0.0 [33].

(3) Indirect constraints from electroweak precision observables such as $\rho_{\ell}, \sin ^{2} \theta_{\text {eff }}^{\ell}$ and $M_{W}$, or their combinations $\epsilon_{i}(i=1,2,3)$ [34]. We require $\epsilon_{i}$ to be compatible with the LEP/SLD data at $95 \%$ confidence level [35]. We also require $R_{b}$ in the NMSSM is within the $2 \sigma$ range of its experimental value. We compute these observables with the formula presented in [36].

For each surviving sample, we further perform a fit using the latest Higgs data presented at the Rencontres de Moriond 2013. These data include the measured signal strengthes for $\gamma \gamma$, $Z Z^{*}, W W^{*}, b \bar{b}$ and $\tau \bar{\tau}$ channels, and their explicit values are summarized in Fig.2 of [5] for the ATLAS results, in Fig.4 of [4] for the CMS results and in Fig.15 of [37] for the CDF+D0 results. We totally use 24 sets of experimental data with 22 of them corresponding to the measured signal strengthes and the other 2 being the combined mass of the Higgs boson reported by the ATLAS and the CMS collaborations respectively. As in our previous works [38], we use the method first introduced in [39] to perform the fit, and properly consider the correlations of the data as in [40, 41]. As will be shown below, the $\chi^{2}$ values in the fit vary from several tens to 170 for the surviving samples of the scan, and in optimal case it may be as low as about 17. In our discussion, we will pay particular attention to the surviving samples with $\chi^{2} \leq 26$. These samples can be used to get the $3 \sigma$ range of any observable $O_{i}$ once they are projected on the $O_{i}$ versus $\delta \chi^{2}$ plane, so hereafter we call them $3 \sigma$ samples (Obviously, the $3 \sigma$ samples are a subset of the surviving samples). For each surviving sample, we also calculate the tuning extent defined by $\Delta=\operatorname{Max}\left\{\left|\partial \ln m_{Z} / \partial \ln p_{i}^{S U S Y}\right|\right\}$ [42], where $p_{i}^{S U S Y}$ denotes a soft breaking parameter at SUSY scale (fixed at $2 \mathrm{TeV}$ in this work).

For the convenience of our analysis, we categorize the surviving samples into three cases according to the nature of the light Higgs scalar (note that a doublet-dominated $h_{1}$ is ruled out by the LEP search for Higgs bosons and $\left.B \rightarrow X_{s} \gamma\right)$ :

- Case A: The light scalar is the CP-odd $A_{1}\left(A_{1}<h / 2\right)$ and it is singlet dominated.

- Case B: The light scalar is the CP-odd $A_{1}\left(A_{1}<h / 2\right)$ and it is doublet dominated.

- Case C: The light scalar is the CP-even $h_{1}\left(h_{1}<h / 2\right)$ and it is singlet dominated. 
TABLE I: The favored parameter ranges for Case A, B and C in the NMSSM. In each item, the range in the first row is for all surviving samples, and the second row corresponds to the $3 \sigma$ samples (the null result means the $3 \sigma$ samples do not exist).

\begin{tabular}{|c|c|c|c|c|c|}
\hline & \multicolumn{2}{|c|}{ Case A } & \multicolumn{2}{|c|}{ Case B } & \multirow{2}{*}{$\frac{\text { Case C }}{h_{2} \text { is SM-like }}$} \\
\hline & $h_{1}$ is SM-like & $h_{2}$ is SM-like & $h_{1}$ is SM-like & $h_{2}$ is SM-like & \\
\hline \multirow{2}{*}{$\lambda$} & $0.1 \sim 0.75$ & $0.23 \sim 0.76$ & $0.1 \sim 0.25$ & $0.1 \sim 0.47$ & $0.20 \sim 0.74$ \\
\hline & $0.1 \sim 0.35$ & $0.23 \sim 0.72$ & - & - & $0.22 \sim 0.74$ \\
\hline \multirow{2}{*}{$\kappa$} & $0.1 \sim 0.65$ & $0.1 \sim 0.25$ & $0.1 \sim 0.54$ & $0.32 \sim 0.6$ & $0.1 \sim 0.46$ \\
\hline & $0.1 \sim 0.63$ & $0.1 \sim 0.23$ & - & - & $0.1 \sim 0.35$ \\
\hline \multirow{2}{*}{$\tan \beta$} & $1.4 \sim 30$ & $1.6 \sim 15$ & $6.5 \sim 12$ & $2.8 \sim 7$ & $1.7 \sim 18$ \\
\hline & $5.2 \sim 30$ & $4.2 \sim 15$ & - & - & $2.8 \sim 16$ \\
\hline \multirow{2}{*}{$\mu(\mathrm{GeV})$} & $170 \sim 1000$ & $108 \sim 270$ & $390 \sim 1000$ & $700 \sim 1000$ & $110 \sim 450$ \\
\hline & $198 \sim 610$ & $115 \sim 235$ & - & - & $110 \sim 262$ \\
\hline \multirow{2}{*}{$M_{A}(\mathrm{GeV})$} & $415 \sim 2000$ & $310 \sim 2000$ & $200 \sim 530$ & $180 \sim 500$ & $370 \sim 2000$ \\
\hline & $850 \sim 2000$ & $580 \sim 2000$ & - & - & $510 \sim 2000$ \\
\hline \multirow{2}{*}{$M_{P}(\mathrm{GeV})$} & $1.3 \sim 160$ & $37 \sim 135$ & $220 \sim 550$ & $1500 \sim 2000$ & $110 \sim 475$ \\
\hline & $10 \sim 80$ & $40 \sim 130$ & - & - & $110 \sim 340$ \\
\hline \multirow{2}{*}{$M_{2}(\mathrm{GeV})$} & $100 \sim 670$ & $290 \sim 1000$ & $100 \sim 700$ & $100 \sim 560$ & $110 \sim 985$ \\
\hline & $110 \sim 560$ & $320 \sim 1000$ & - & - & $160 \sim 965$ \\
\hline \multirow{2}{*}{$M_{Q_{3}}(\mathrm{GeV})$} & $205 \sim 2000$ & $215 \sim 2000$ & $280 \sim 2000$ & $100 \sim 1500$ & $505 \sim 2000$ \\
\hline & $345 \sim 2000$ & $330 \sim 2000$ & - & - & $585 \sim 1980$ \\
\hline \multirow{2}{*}{$M_{U_{3}}(\mathrm{GeV})$} & $180 \sim 2000$ & $400 \sim 2000$ & $200 \sim 2000$ & $100 \sim 2000$ & $500 \sim 2000$ \\
\hline & $235 \sim 2000$ & $400 \sim 2000$ & - & - & $570 \sim 2000$ \\
\hline \multirow{2}{*}{$A_{t}(\mathrm{GeV})$} & $-4960 \sim 4920$ & $-5000 \sim 5000$ & $-5000 \sim-2000$ & $-3000 \sim-300$ & $-4960 \sim 4980$ \\
\hline & $-4400 \sim 4630$ & $-5000 \sim 5000$ & - & - & $-4960 \sim 4870$ \\
\hline \multirow{2}{*}{$M_{\tilde{l}}(\mathrm{GeV})$} & $100 \sim 1000$ & $100 \sim 500$ & $100 \sim 750$ & $100 \sim 350$ & $100 \sim 800$ \\
\hline & $100 \sim 1000$ & $100 \sim 500$ & - & - & $100 \sim 800$ \\
\hline \multirow{2}{*}{$A_{\lambda}(\mathrm{GeV})$} & $-2500 \sim 1920$ & $-550 \sim 2180$ & $-2000 \sim-400$ & $-3700 \sim-800$ & $300 \sim 2150$ \\
\hline & $-600 \sim 820$ & $-550 \sim 2100$ & - & - & $465 \sim 2000$ \\
\hline \multirow{2}{*}{$A_{\kappa}(\mathrm{GeV})$} & $-34 \sim 75$ & $-38 \sim 61$ & $-70 \sim-16$ & $-1300 \sim-200$ & $-620 \sim-70$ \\
\hline & $-5 \sim 0.16$ & $-35 \sim 35$ & - & - & $-395 \sim-70$ \\
\hline
\end{tabular}



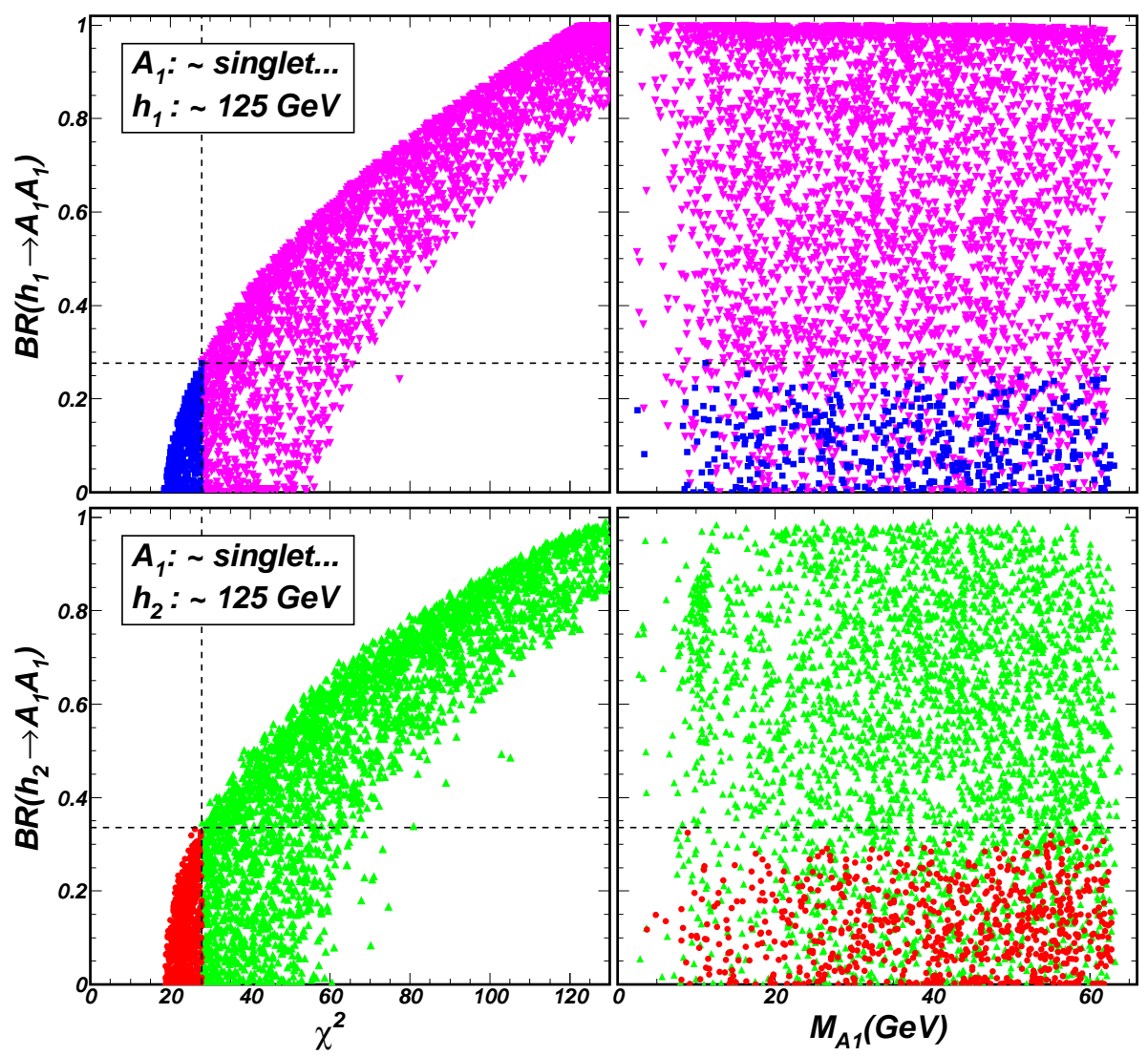

FIG. 1: The scatter plots of the surviving samples in Case A projected on the plane of $\chi^{2}$ versus $\operatorname{Br}\left(h \rightarrow A_{1} A_{1}\right)(h$ denotes the SM-like Higgs boson $)$ and the plane of $m_{A_{1}}$ versus $\operatorname{Br}\left(h \rightarrow A_{1} A_{1}\right)$ respectively. The upper panel is for the 'SM-like $h_{1}$ ' scenario with the $3 \sigma$ samples marked out as squares (blue), and the bottom panel is for the the 'SM-like $h_{2}$ ' scenario with the $3 \sigma$ samples marked out as circles (red).

\section{A. Case A $\left(A_{1}<h / 2\right.$, singlet dominated $)$}

In Case A, the SM-like $125 \mathrm{GeV}$ Higgs boson $h$ may be either the lightest CP-even state $h_{1}$ or the next-to-lightest CP-even state $h_{2}$. In Table I, we list the favored parameter ranges for all the surviving samples and the $3 \sigma$ samples in Case A. We note that in this case the parameter $\tan \beta$ can be very large [43]. This table indicates that in each scenario the ranges of some parameters for the surviving samples are significantly wider than the corresponding $3 \sigma$ samples. Furthermore, we compare the number of all the surviving samples with the $3 \sigma$ samples, and find that the latter is at most one fifth of the former. These facts reflect that the current LHC Higgs data can severely constrain the parameter space of the NMSSM. This table also indicates that, in order to predict a light singlet-dominated $A_{1}$, the value of 


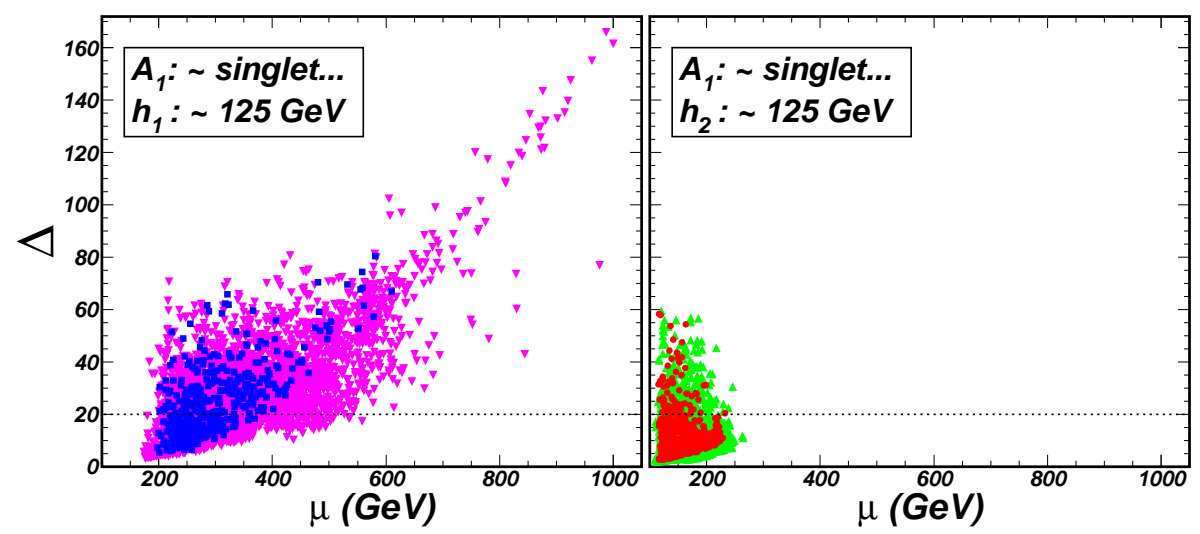

FIG. 2: Same as Fig, 1, but projected on the $\mu$ versus $\Delta$ plane.

$M_{P}$ should be less than $160 \mathrm{GeV}$.

From analyzing the surviving samples, we find two features for Case A:

- One is that the $\chi^{2}$ value in the fit of the Higgs data may be rather low with $\chi_{\min }^{2} \simeq 17$ for 24 sets of experimental data, and it increases as the branching ratio of the exotic decay $h \rightarrow A_{1} A_{1}$ becomes larger. This feature is exhibited in Fig.1. This figure reflects the fact that the NMSSM can explain the Higgs data quite well given that $\operatorname{Br}\left(h \rightarrow A_{1} A_{1}\right)$ is moderately small. This figure also reveals the information that, without the Higgs data, $\operatorname{Br}\left(h \rightarrow A_{1} A_{1}\right)$ can exceed 90\%, while after considering the constraints from the Higgs data at $3 \sigma$, it is less than $28 \%$ for $h_{1}$ being the SM-like Higgs (the 'SM-like $h_{1}$ ' scenario) and $34 \%$ for $h_{2}$ being the SM-like Higgs (the 'SM-like $h_{2}$ ' scenario). This conclusion is independent of the value of $m_{A_{1}}$. As a comparison, we checked that for any exotic decays of the Higgs boson (with the SM Higgs couplings to fermions and gauge bosons), the Higgs data restrain the exotic decay branching ratio to be less than $28 \%$ at $3 \sigma$ level. This result can be seen as an update of that in [44] after the Rencontres de Moriond 2013, but different from those in [45] for different data treatments.

- The other feature is that the tuning extent $\Delta$ can be less than 10 , reflecting that the NMSSM is quite natural. This feature is shown in Fig, 2 . Compared with the 'SM-like $h_{1}$ ' scenario, a lower $\Delta$ is predicted for the 'SM-like $h_{2}$ ' scenario. This is because $m_{Z}$ is sensitive to the value of $\mu$ (note the tree level relation $m_{Z}^{2}=2\left(m_{H_{d}}^{2}-\right.$ $\left.m_{H_{u}}^{2} \tan ^{2} \beta\right) /\left(\tan ^{2} \beta-1\right)-2 \mu^{2}$ with $m_{H_{d}}^{2}$ and $m_{H_{u}}^{2}$ representing the weak scale soft SUSY breaking masses of the Higgs fields [42]), and for the 'SM-like $h_{2}$ ' scenario a 


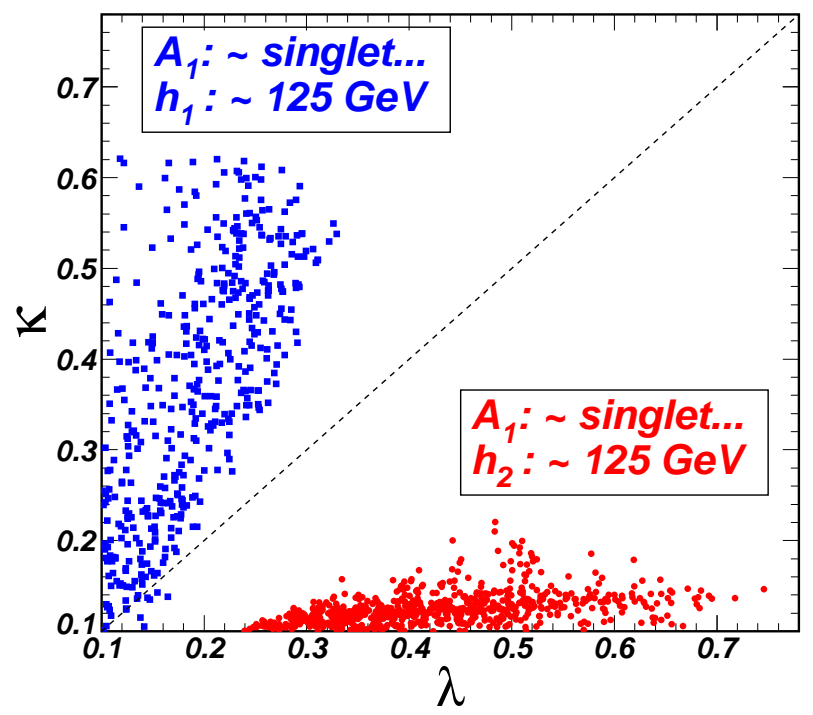

FIG. 3: Same as Fig,1, but projected on the $\lambda$ versus $\kappa$ plane (here only the $3 \sigma$ samples are ploted).

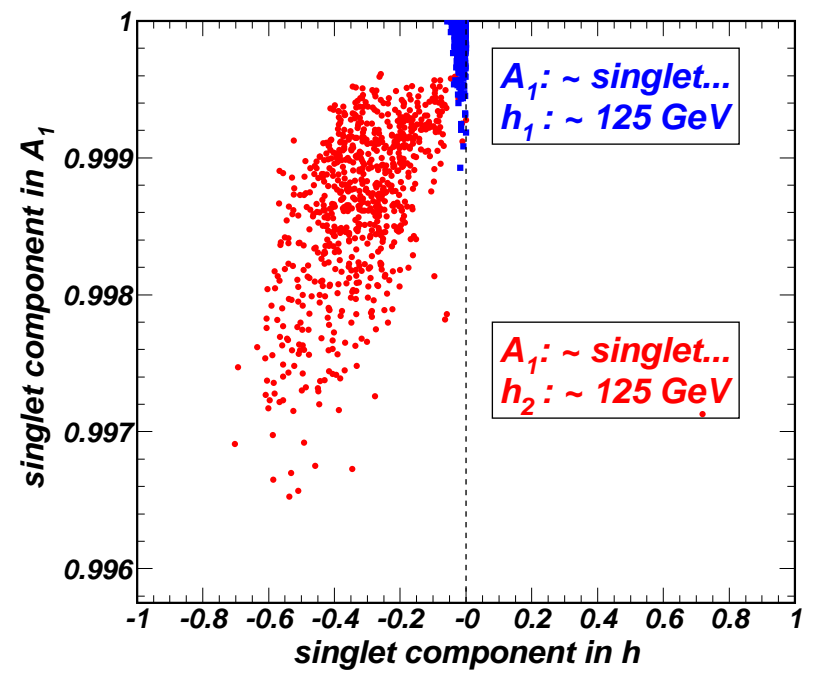

FIG. 4: Same as Fig 3, but show the singlet component coefficients of $A_{1}$ and $h$.

lower $\mu$ is preferred.

About Case A, more points should be noted. (i) The first is that the 'SM-like $h_{1}$ ' scenario and the 'SM-like $h_{2}$ ' scenario actually correspond to two distinct parameter regions of the NMSSM. To illustrate this point, we consider the parameters $\lambda$ and $\kappa$ and project the $3 \sigma$ samples on the $\lambda$ versus $\kappa$ plane in Fig, This figure indicates that, in contrast with the fact that most samples for the 'SM-like $h_{1}$ ' scenario satisfy $\lambda \lesssim \kappa$, the 'SM-like $h_{2}$ ' scenario is characterized by $\lambda \gg \kappa$. The reason is that as far as the $3 \sigma$ samples are concerned, $M_{33}$ in Eq.([6) is approximated by $M_{33} \simeq 4\left(\kappa v_{s}\right)^{2}=4(\kappa \mu / \lambda)^{2}$. Given $\mu>100 \mathrm{GeV}$ as required by the LEP bound on chargino mass, $\lambda$ should be much larger than $\kappa$ to guarantee $M_{22}^{2}>M_{33}^{2}$, 

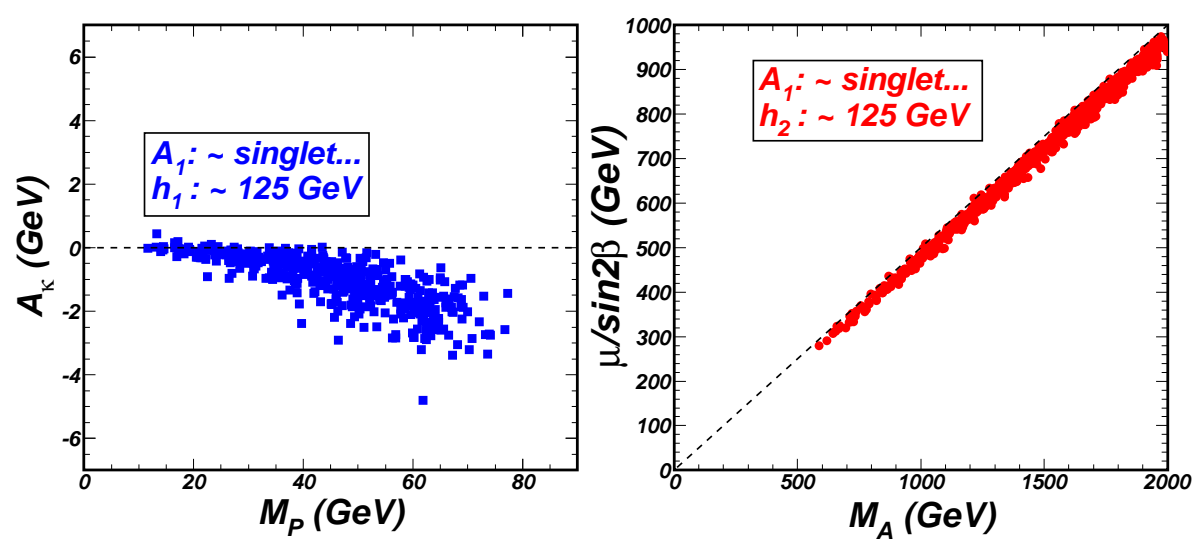

FIG. 5: Same as Fig 3, but showing the correlation of $M_{P}$ with $A_{\kappa}$ for the 'SM-like $h_{1}$ ' scenario (left panel) and the correlation of $M_{A}$ with $\mu / \sin 2 \beta$ for the 'SM-like $h_{2}$ ' scenario (right panel).

which is a necessary condition to predict $h_{2} \sim 125 \mathrm{GeV}$. (ii) The second point is that $A_{1}$ should be highly singlet dominated and the properties of the SM-like Higgs boson for the 'SM-like $h_{1}$ ' scenario and the 'SM-like $h_{2}$ ' scenario may be quite different. To exhibit this conclusion, we show in Fig 4 the singlet component coefficients of $A_{1}$ and $h$ for the $3 \sigma$ samples. This figure indicates that the singlet component coefficient of $A_{1}$ (i.e. $U_{12}$ ) is larger than 0.99 for both scenarios. This figure also indicates that the SM-like $h_{1}$ has a very small singlet component (i.e. $V_{13} \sim 1 \%$ ) while the SM-like $h_{2}$ may have a sizable singlet component with the corresponding coefficient $V_{23}$ reaching 0.7 . In fact, we checked that the $h b \bar{b}$ coupling is approximately equal to the SM value for the 'SM-like $h_{1}$ ' scenario and may be much smaller for the 'SM-like $h_{2}$ ' scenario. About Case A, we remind that, due to the singlet nature of $A_{1}$, the $h A_{1} A_{1}$ interaction should be very weak, but on the other hand, since the total width of the SM-like Higgs boson is also small (about $4 \mathrm{MeV}$ in the $\mathrm{SM}), \operatorname{Br}\left(h \rightarrow A_{1} A_{1}\right)$ may still be sizable. (iii) The last point is that, since we require the theory to predict a light scalar and meanwhile satisfy various experimental constraints, some parameters are limited in certain narrow ranges or correlate with other parameters, as shown in Fig.5. The left panel indicates that in the 'SM-like $h_{1}$ ' scenario we have $A_{\kappa} \simeq 0$, and the right panel shows that in the 'SM-like $h_{2}$ ' scenario we have $M_{A} \sin 2 \beta / \mu \simeq 2$. We checked that a very small $A_{\kappa}$ is needed to predict a light singlet dominated $A_{1}$, while the correlation $M_{A} \sin 2 \beta / \mu \simeq 2$ is characteristic in predicting $h_{2} \simeq 125 \mathrm{GeV}$, as observed in [6]. 


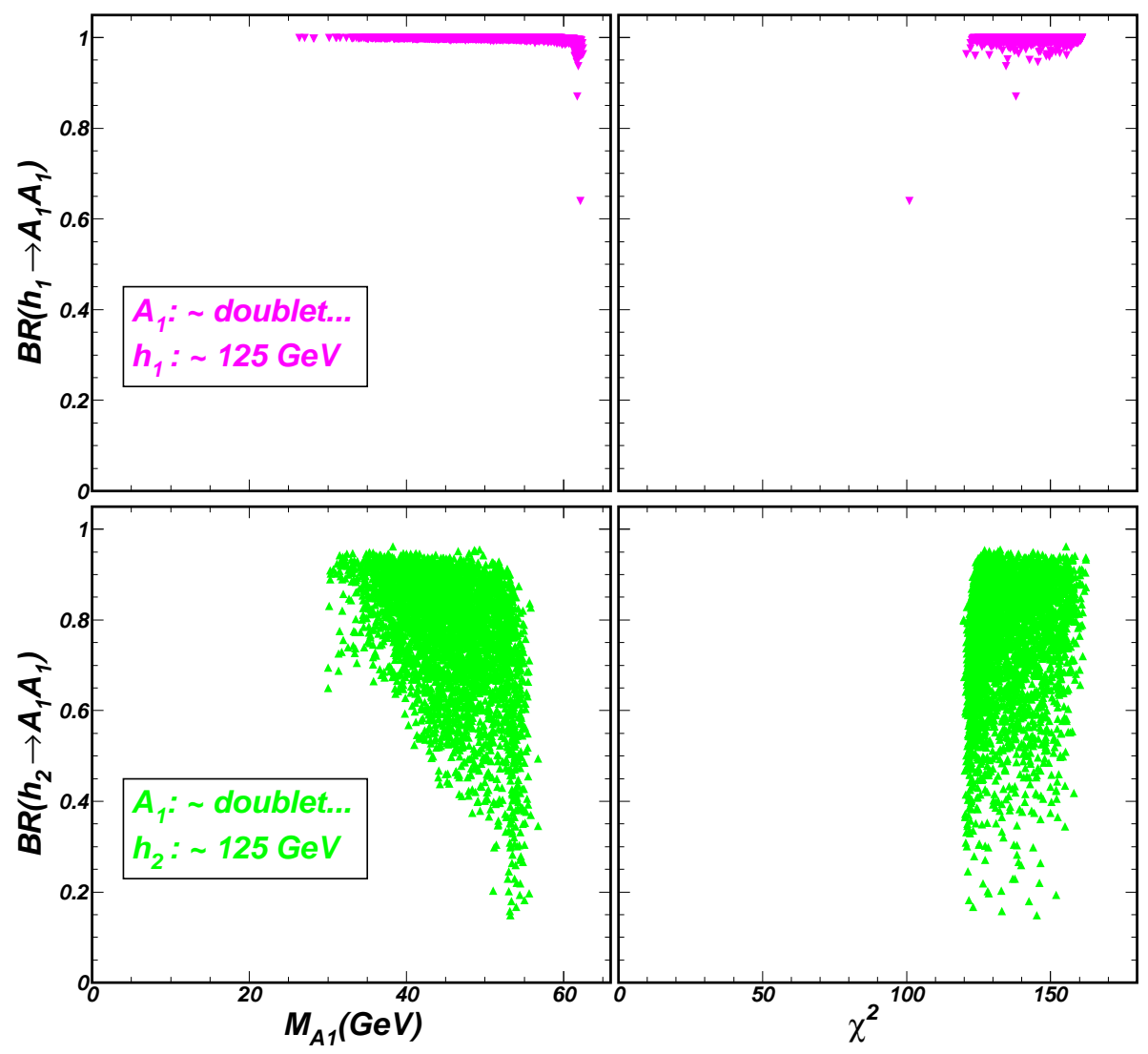

FIG. 6: Same as Fig 1, but showing the surviving samples in Case B (no $3 \sigma$ samples).
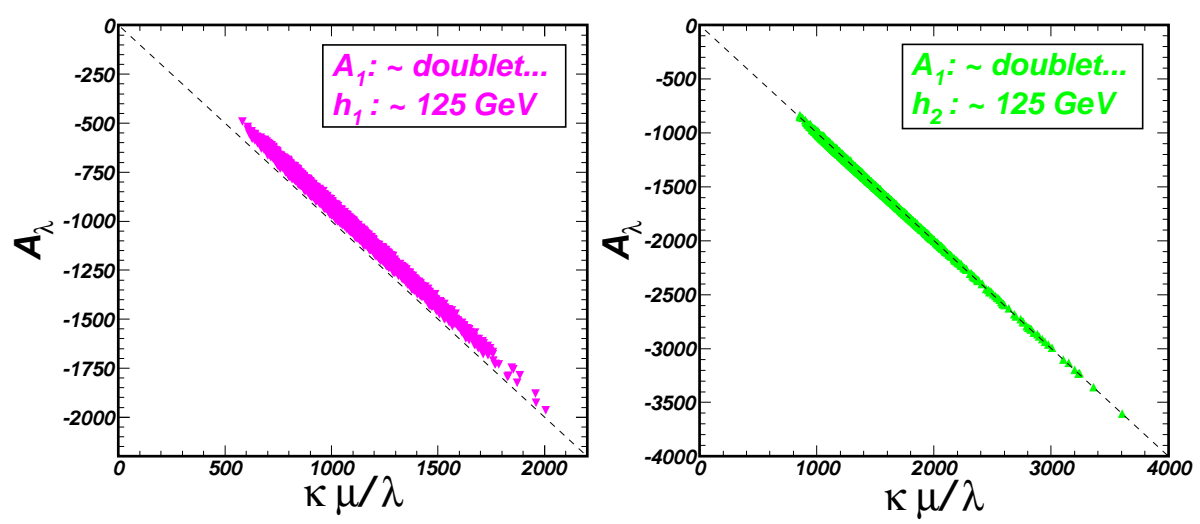

FIG. 7: Same as Fig,5, but showing the correlation of $\kappa \mu / \lambda$ with $A_{\lambda}$ for the surviving samples in Case B.

\section{B. Case B $\left(A_{1}<h / 2\right.$, doublet dominated $)$}

As in Case A, the SM-like $125 \mathrm{GeV}$ Higgs boson in Case B may be either $h_{1}$ or $h_{2}$, and the corresponding favored parameter regions of the surviving samples are shown in Table I. We emphasize that the parameter $M_{A}$ in this table is defined at the scale of $2 \mathrm{TeV}$, and in 


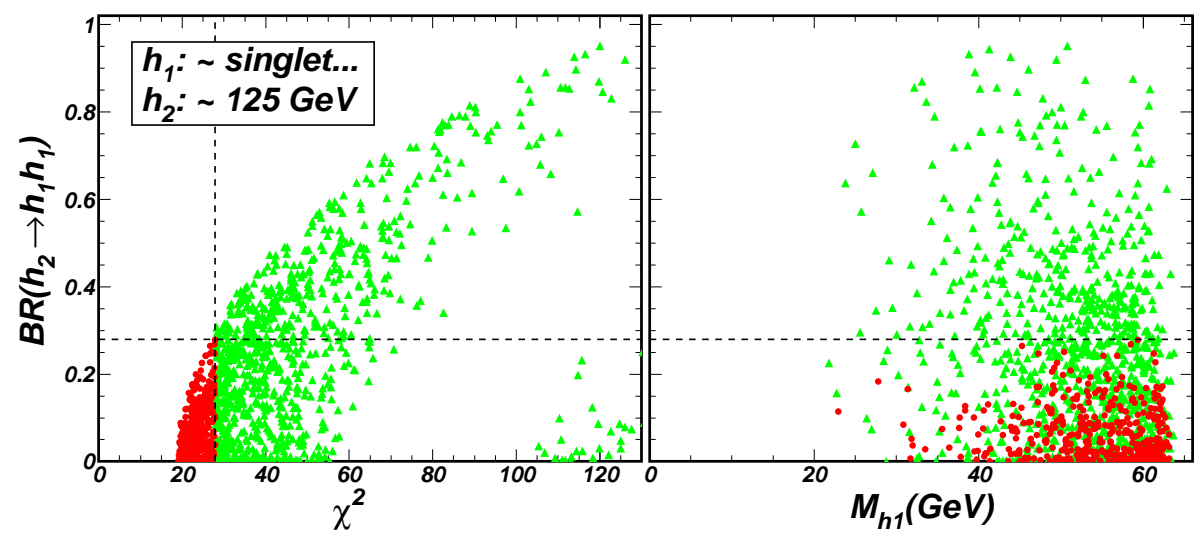

FIG. 8: Same as Fig!1, but for Case $\mathrm{C}$ where $h_{2}$ is the SM-like Higgs boson.

calculating the CP-odd Higgs boson masses by the NMSSMTools we use the value at the mass scale of the third generation squarks which can be obtained by the renormalization group equation. Moreover, we checked that the surviving samples are characterized by a relatively large matrix element $M_{-12}^{2}$ in Eq.(7). This is helpful to suppress the mass of $A_{1}$.

In Fig [6 we project the surviving samples on the plane of $M_{A_{1}}$ versus $\operatorname{Br}\left(h \rightarrow A_{1} A_{1}\right)$ and the plane of $\chi^{2}$ versus $\operatorname{Br}\left(h \rightarrow A_{1} A_{1}\right)$ respectively. This figure indicates that in the 'SM-like $h_{1}$ ' scenario, the branching ratio of the decay $h \rightarrow A_{1} A_{1}$ is always larger than $60 \%$ so that $\chi^{2}>100$, while in the 'SM-like $h_{2}$ ' scenario, although the rate of the decay $h \rightarrow A_{1} A_{1}$ may be small, e.g. about $10 \%$ for $m_{A_{1}} \simeq 55 \mathrm{GeV}$, the $\chi^{2}$ value is still larger than 100 . The reason is that the $h b \bar{b}$ coupling in the 'SM-like $h_{2}$ ' scenario is at least one times larger than its SM prediction. In fact, the 'SM-like $h_{2}$ ' scenario in Case B actually corresponds to a non-decoupling region of the NMSSM since the mass of the charged Higgs boson varies from $130 \mathrm{GeV}$ to $150 \mathrm{GeV}$. Consequently, the properties of the SM-like Higgs boson are expected to deviate greatly from the SM prediction. To summarize, Fig 6 indicates that Case B is actually disfavored by the fit of the Higg data (no $3 \sigma$ samples exist).

Also as in Case A, a strong correlation between some parameters is needed to predict a doublet dominated $A_{1}$. In Fig:7 we show the correlation between the parameter $A_{\lambda}$ and the parameter $\kappa \mu / \lambda$ for the surviving samples in this case. From Eq.(7), one can infer that such a correlation is needed to reduce the value of $M_{A}$. 


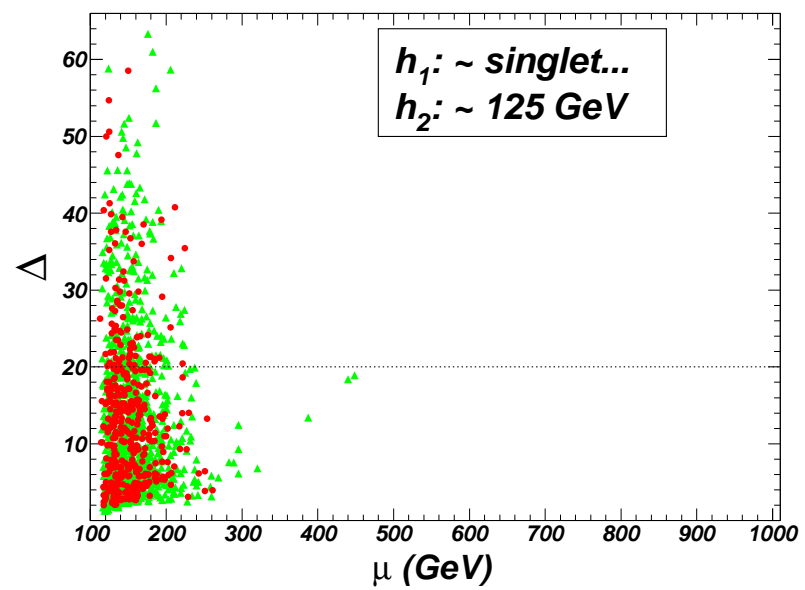

FIG. 9: Same as Fig,2, but for Case C.

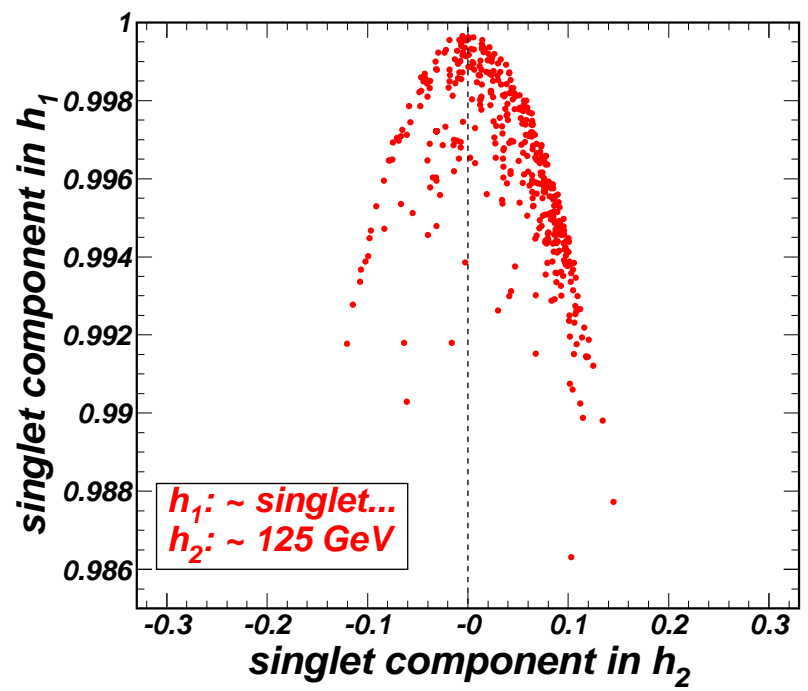

FIG. 10: Same as Fig, 4 , but for the singlet component coefficients for $h_{1}$ and $h_{2}$ in Case C.

\section{Case $\mathbf{C}\left(h_{1}<h / 2\right.$, singlet dominated $)$}

In Case $\mathrm{C}$ the SM-like Higgs boson is the next-to-lightest CP-even state $h_{2}$, and due to the strong constraints from the LEP search for Higgs bosons and $B \rightarrow X_{s} \gamma$, a doublet dominated $h_{1}$ is actually ruled out. In Table I, we show the favored parameter regions for the surviving samples and also the $3 \sigma$ samples. As pointed out in [21], in order to predict a light $h_{1}$, one only needs to tune the value of $A_{\kappa}$ when other parameters are fixed. So, except for the correlation shown on the left panel of Fig 5 and the condition $\kappa<\lambda$ which is necessary to predict $m_{h_{2}} \simeq 125 \mathrm{GeV}$, there is no other special features for the parameters of Case C.

Like the 'SM-like $h_{2}$ ' scenario in Case A, the $\chi^{2}$ value and the parameter $\Delta$ may be as 
low as about 17 and 10 respectively. These features are presented in Fig, 8 and Fig, About Case $\mathrm{C}$, one should note that the branching ratio of $h \rightarrow h_{1} h_{1}$ should be less than $28 \%$ at $3 \sigma$ level (see Fig,8). One should also note that, as shown in Fig,10 where the singlet component coefficients of $h_{1}$ and $h_{2}$ are presented for the $3 \sigma$ samples, $h_{1}$ in Case $\mathrm{C}$ is highly singlet dominated while $h_{2}$ is highly doublet dominated.

In summary, one may conclude that the current experiments still allow for the existence of a light scalar (CP-even or CP-odd). But the LHC Higgs data have required it to be highly singlet dominated. Moreover, in the NMSSM either $h_{1}$ or $h_{2}$ may play the role of the SM-like Higgs boson $h$, and for each case the properties of $h$ may be quite different.

\section{DETECTION OF A LIGHT SCALAR AT FUTURE COLLIDERS}

As discussed in the preceding section, if there exists a light scalar with mass lighter than half the SM-like Higgs boson mass in the NMSSM, it should be highly singlet dominated. Consequently, its interactions with the fermions and the gauge bosons in the SM are very weak, which implies that this scalar is difficult to search at colliders. But on the other hand, although the interaction of this scalar with the SM-like Higgs boson is also weak, the rate of $h$ decay into the scalar pair may still be sizable due to the narrow width of $h$. This fact motivates us to scrutinize the decay product of $h$ to search for the light scalar. In the following, we take Case A as an example to discuss the prospect of such a search via different processes at colliders.

First, we consider the light $A_{1}$ comes from the $Z$-decay. For this end, we calculate the branching ratios of the rare decays $Z \rightarrow A_{1} b \bar{b}$ and $Z \rightarrow A_{1} \gamma$ with the code of our previous work [46] and show these ratios in Fig.11. This figure indicates that, as far as the $3 \sigma$ samples in Case $\mathrm{A}$ are concerned, the ratios are at most $10^{-8}$ and $10^{-12}$, respectively. Since the dominant decay product of $A_{1}$ is $b \bar{b}$ with a branching ratio being about $90 \%$, the main signals of the decays are $b \bar{b} b \bar{b}$ and $b \bar{b} \gamma$, respectively. Then, compared with the LEP uncertainties on these signals, we learn that the ratios are at least $10^{-4}$ lower than the LEP sensitivity [25].

Second, we consider the $h A_{1}$ associated production at an electron-positron collider with $\sqrt{s}=250 \mathrm{GeV}$. In Fig 12 we show the production rate as a function of $m_{A_{1}}$. Obviously, since the rate is maximally at the order of $10^{-3} \mathrm{fb}$, this associated production process can 

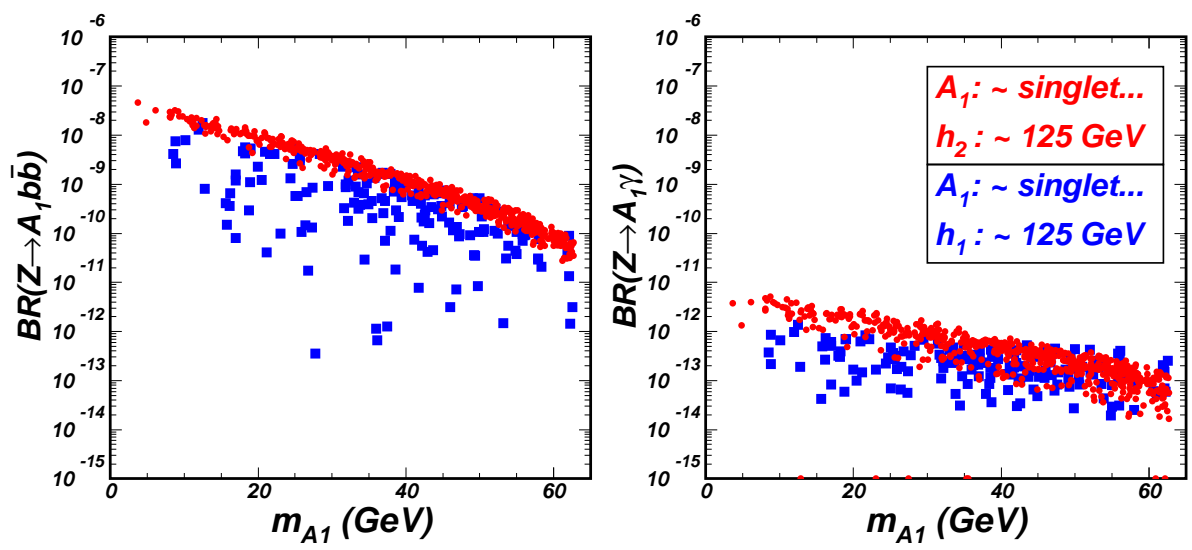

FIG. 11: The branching ratios of the rare $Z$ decays $Z \rightarrow A_{1} b \bar{b}$ and $Z \rightarrow A_{1} \gamma$ as a function of $m_{A_{1}}$ for the $3 \sigma$ samples in Case A. The squares (blue) correspond to the results of the 'SM-like $h_{1}$ ' scenario, and the bullets (red) are for the 'SM-like $h_{2}$ ' scenario.

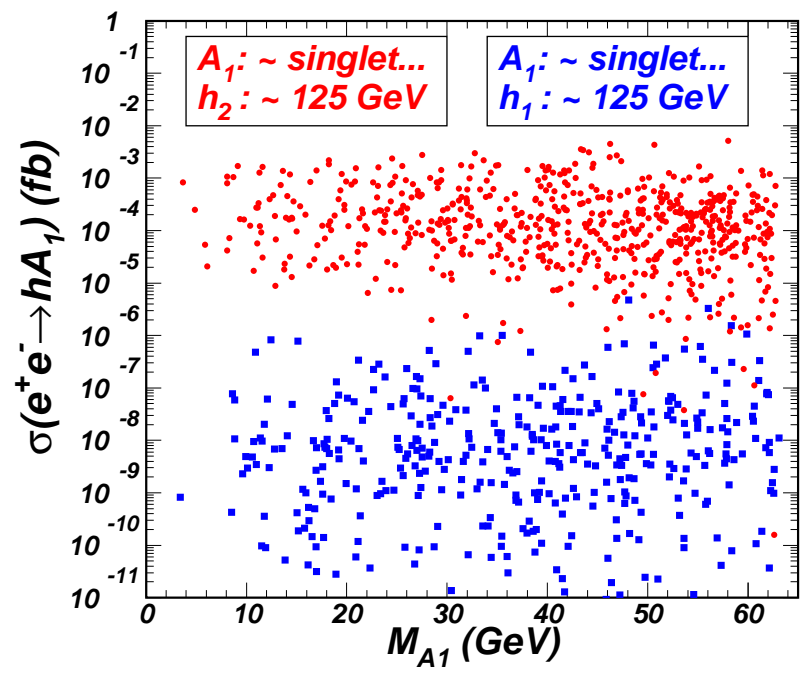

FIG. 12: Same as Fig 11, but for the cross section of $h A_{1}$ associated production at an electronpositron collider with $\sqrt{s}=250 \mathrm{GeV}$.

hardly be utilized to search for the scalar.

Next we investigate the possibility of searching for $A_{1}$ at the LHC via the decay $h \rightarrow$ $A_{1} A_{1} \rightarrow 4 b$. Such an issue has been discussed in [47, 48] and it was found that the process $p p \rightarrow V h \rightarrow l+4 b+X(V=W, Z, l$ denotes one lepton and $X$ denotes anything) is well suited for such a search. In this work, we fix $m_{h}=125 \mathrm{GeV}$ and perform an analysis as in [47]. The signal contains at least one isolated lepton, $e$ or $\mu$, and exactly $4 b$-tagged jets. The corresponding backgrounds mainly come from the $t \bar{t}$ production with one top quark decaying hadronically and the other top quark decaying semi-leptonically, the $t \bar{t} b \bar{b}$ production with 
TABLE II: The rates of the signal and various backgrounds after different cuts for $m_{A_{1}}=45 \mathrm{GeV}$ and $C_{4 b}^{2}=0.33$.

\begin{tabular}{llllllllll}
\hline \hline & $t \bar{t}$ & $t \bar{t}+b \bar{b}$ & $t \bar{t}+c \bar{c}$ & $\mathrm{~V}+$ jets & Total bkg & $\mathrm{Zh}$ & $W^{+} h$ & $W^{-} h$ & Total signal \\
\hline$\sigma_{\text {basic cuts }}(f b)$ & 12.45 & 3.28 & 0.039 & 0.264 & 16.13 & 0.049 & 0.133 & 0.087 & 0.26 \\
$\sigma_{M_{4 b} \text { cut }}(f b)$ & 0.170 & 0.031 & 0.00045 & 0.016 & 0.22 & 0.024 & 0.095 & 0.053 & 0.17 \\
\hline \hline
\end{tabular}

some of the top quark decay products missed, the $t \bar{t} c \bar{c}$ production with the charm quark jets mistagged as bottom quark jets and also the $W / Z+4 b$ production processes. In our simulation, the signal and background processes are modeled with MadGraph 5 [49], which incudes Pythia 6.4 [50] for initial and final state radiation, parton shower and hadronization, and pass through the fast detector simulation with DELPHES [51]. Jets are reconstructed with FastJet [52, 53] by using the anti- $k_{T}$ algorithm with a distance parameter of 0.5 . The cuts we considered are:

- The basic cuts:

$$
\begin{aligned}
& p_{T}(j) \geq 15 \mathrm{GeV}, \quad|\eta(j)| \leq 2.5, \quad p_{T}(l) \geq 15 \mathrm{GeV}, \quad|\eta(l)| \leq 2.5 \\
& \Delta R(b, b) \geq 0.4, \quad \Delta R(b, l) \geq 0.4
\end{aligned}
$$

where $p_{T}$ denotes the transverse momentum, $\eta$ represents pseudorapidity and $\Delta R(b, j)=\sqrt{(\Delta \eta)^{2}+(\Delta \phi)^{2}}$ is the angular separation of the b-jet and the particle $j(j=b, l)$.

- $\left|M_{4 b}-115\right| \leq 15 \mathrm{GeV}$ with $M_{4 b}$ denoting the invariant mass of the four bottom quarks. This cut is motivated by the fact that the four bottom quarks originate from the SM-like Higgs boson decay, and due to possible momentum missing in the jet reconstruction, $M_{4 b}$ is peaked at about $115 \mathrm{GeV}$ instead of at the Higgs boson mass [54].

Moreover, in order to get a realistic estimation of the signal and backgrounds, we also assume a b-tagging efficiency of $70 \%$ for a bottom quark jet and a mis-tagging probability of $5 \%$ (1\%) for a charm quark jet (light quark or gluon jet).

Noticing that the signal rate after the cuts depends only on an overall scaling factor

$$
C_{4 b}^{2}=\left(\frac{g_{V V h}^{N M S M}}{g_{V V h}^{S M}}\right)^{2} \times B r\left(h \rightarrow A_{1} A_{1}\right) \times\left(B r\left(A_{1} \rightarrow b \bar{b}\right)\right)^{2},
$$




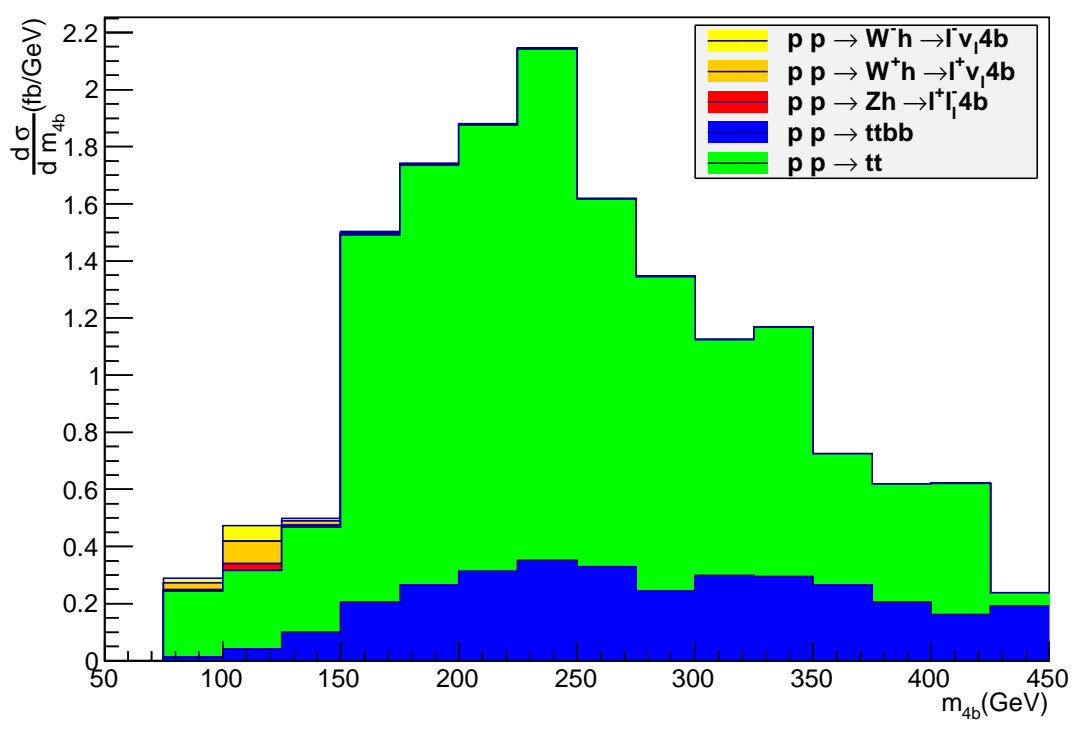

FIG. 13: The invariant mass $M_{4 b}$ distribution for the signal and the backgrounds after the basic cuts. Here we fix $m_{A_{1}}=45 \mathrm{GeV}$ and $C_{4 b}^{2}=0.33$.

which determines the cross section of the process $p p \rightarrow V h \rightarrow V 4 b$ at the LHC, and the mass of $A_{1}$ which determines the cut efficiency, we fix $m_{A_{1}}=45 \mathrm{GeV}$ and $C_{4 b}^{2}=0.33$, and illustrate the distributions of $M_{4 b}$ for both the signal and various backgrounds in Fig $[13$, We also list the rates of the signal and the backgrounds after different cuts in Table II. These results indicate that the $M_{4 b}$ cut is very efficient in suppressing the backgrounds, and also that the $t \bar{t}$ background is still dominant over other backgrounds after the cut. Moreover, for the benchmark point we considered, we estimate that its significance $S / \sqrt{B}$ is about 6.37 for an integrated luminosity of $300 \mathrm{fb}^{-1}$.

In order to exhibit the capability of the LHC in the $A_{1}$ search, in Fig 14 we plot the $3 \sigma$ samples together with the significance curves of $S / \sqrt{B}=2,3,5$ for an luminosity of $300 \mathrm{fb}^{-1}$ on the $m_{A_{1}}$ versus $C_{4 b}^{2}$ plane. This figure shows that in order to discover the light scalar, $C_{4 b}^{2}$ should be larger than 1 for $m_{A_{1}} \lesssim 25 \mathrm{GeV}$, and with the increase of $m_{A_{1}}$, the requirement on $C_{4 b}^{2}$ decreases to 0.2 for $m_{A_{1}}=60 \mathrm{GeV}$. We can also see that nearly all of the $3 \sigma$ samples in the two scenarios are under the $S / \sqrt{B}=5$ curve, which means that in order to discover the light scalar a luminosity over $300 \mathrm{fb}^{-1}$ is needed.

Compared with the simulation result in [47], we note our significance is much lower. The reason is that the authors of [47] performed the simulation at parton level, while in our analyse we considered the initial and final state radiation, the parton shower and the 


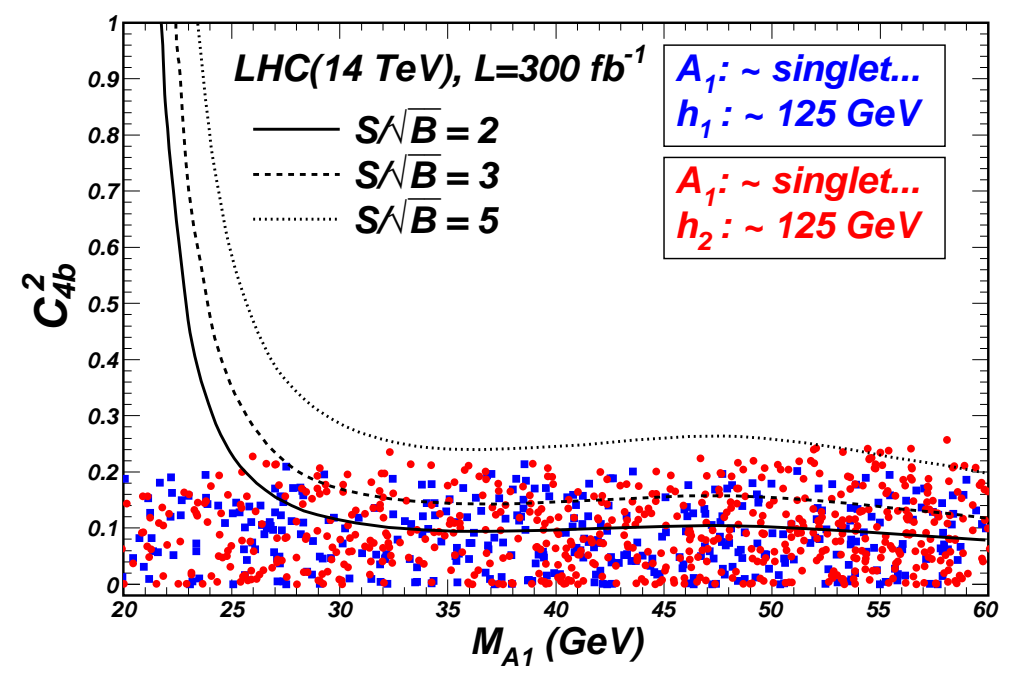

FIG. 14: Same as Fig.11, but projected on the plane of $M_{A_{1}}$ versus $C_{4 b}^{2}$. The significance of the LHC-14 for $300 \mathrm{fb}^{-1}$ integrated luminosity is also plotted on this plane.

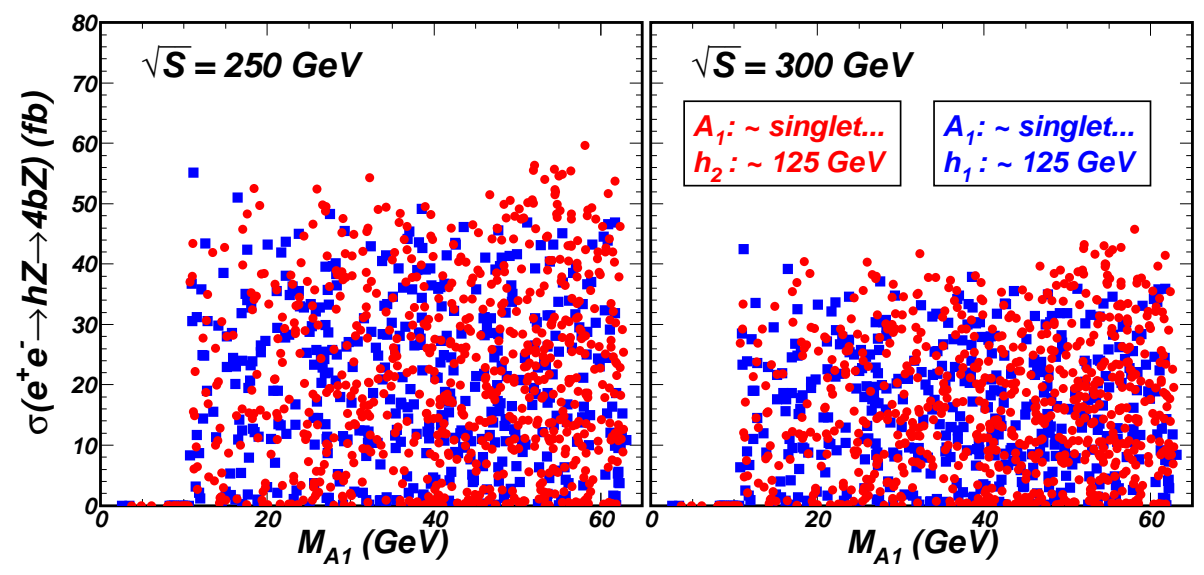

FIG. 15: Same as Fig[12, but for the cross section of the process $e^{+} e^{-} \rightarrow Z h \rightarrow Z 4 b$ at an electron-positron collider with $\sqrt{s}=250 \mathrm{GeV}$ and $300 \mathrm{GeV}$ respectively.

hadronization effect with Pythia, the detector effect with DELPHES, and the reconstruction of jets with FastJet. Consequently, the $M_{4 b}$ distribution of the $t \bar{t}$ production moves towards lower end so that the $t \bar{t}$ production is still the dominant background after the cuts. This is quite different from the results of [47] where the main background comes from the $t \bar{t} b \bar{b}$ production. Another consequence of our treatment is that the jet reconstruction can hurt both the signal and the backgrounds greatly, especially for our case where the signal contains exactly four b-jets. We checked that if we perform the simulation at parton level as in [47], we can reproduce its results.

Finally, since the properties of $h$ can be precisely measured though the $Z h$ associated 
production at an electron-positron collider, we also calculate the cross section of the process $e^{+} e^{-} \rightarrow Z h \rightarrow Z 4 b$ for a collision energy $\sqrt{s}=250 \mathrm{GeV}$ and $300 \mathrm{GeV}$ respectively. The results are shown in Fig,15. This figure indicates that, as far as the $3 \sigma$ samples in Case A are concerned, the rate can be as large as $56 \mathrm{fb}$ for $\sqrt{s}=250 \mathrm{GeV}$. Compared with the same final state at the LHC with $\sqrt{s}=14 \mathrm{TeV}$, although such a production rate is only about one fourth, the signal is free of the backgrounds listed in Table II. So a rather low prodcution rate at an electron-positron collider may result in the $A_{1}$ discovery. We checked that a production rate over $10 \mathrm{fb}$ corresponds to $C_{4 b}^{2}>0.04$ (such a small $C_{4 b}^{2}$ is not accessible at the LHC for $300 \mathrm{fb}^{-1}$ integrated luminosity). Fig 15 also indicates that, since the $Z h$ associated production is a $s$-channel process, the signal rate decreases as the increase of the collision energy.

\section{CONCLUSION}

In the NMSSM, due to the introduction of one new gauge singlet Higgs field, one of the neutral Higgs scalars (CP-even or CP-odd) may be lighter than half the SM-like Higgs boson. In this case, the SM-like Higgs boson $h$ can decay into the scalar pair and consequently the visible $\gamma \gamma$ and $Z Z^{*}$ signal rates at the LHC will be suppressed. In this work, we checked the constraints of the latest LHC Higgs data on such a possibility. First, we scanned comprehensively the parameter space of the NMSSM by considering various experimental constraints. Then we focused on the surviving samples which predict a light scalar. According to the properties of the scalar, we categorized the samples into three cases calsses: Case A $\left(A_{1}<h / 2\right.$, singlet dominated), Case B $\left(A_{1}<h / 2\right.$, doublet dominated $)$ and Case $\mathrm{C}\left(h_{1}<h / 2\right.$, singlet dominated). For the surviving samples we performed a fit using the latest LHC Higgs data. We found that the Higgs data can severely constrain the parameter space, e.g., for Case A and Case C, less than one fifth of the surviving samples are allowed by the Higgs data at $3 \sigma$ level, and for Case B all samples are actually ruled out. We further focused on the $3 \sigma$ samples allowed by the Higgs data and analysed the properties of the light scalar, including its favored parameter region, its composition as well as the ratio of $h$ decay into the scalar pair. Finally, we examined the detection of such a scalar at future colliders. From our analysis we obtained the following observations:

(i) Without the LHC Higgs data, the light Higgs boson $A_{1}$ can be either singlet-dominated 
or doublet-dominated; while after considering the constraints from the Higgs data, it should be highly singlet dominated.

(ii) In the 'SM-like $h_{1}$ ' and 'SM-like $h_{2}$ ' scenarios of Case A, the Higgs data require the branching ratio of $h \rightarrow A_{1} A_{1}$ to be less than $28 \%$ and $34 \%$ respectively; while in the 'SM-like $h_{2}$ ' scenario of Case C, the Higgs data require the ratio of $h \rightarrow h_{1} h_{1}$ to be less than $28 \%$.

(iii) An efficient way at the LHC to detect the light scalar is through the $V h(V=W, Z)$ associated production with $h$ decaying exotically into four bottom quarks. A detailed Monte Carlo simulation indicates that, if the branching ratio of the exotic decay is less than $30 \%$, more than $300 \mathrm{fb}^{-1}$ luminosity is needed to discover the scalar. At a future electron-positron collider with $\sqrt{s} \simeq 250 \mathrm{GeV}$, the capability to detect the light scalar may be greatly improved by looking for the process $e^{+} e^{-} \rightarrow Z h \rightarrow Z A_{1} A_{1} \rightarrow Z 4 b$.

\section{Acknowledgement}

This work was supported in part by the National Natural Science Foundation of China (NNSFC) under grant Nos. 10821504, 11135003, 10775039, 11075045, by Specialized Research Fund for the Doctoral Program of Higher Education with grant No. 20104104110001, and by the Project of Knowledge Innovation Program (PKIP) of Chinese Academy of Sciences under grant No. KJCX2.YW.W10.

[1] G. Aad et al. [ATLAS Collaboration], Phys. Lett. B 716 (2012) 1.

[2] S. Chatrchyan et al. [CMS Collaboration], Phys. Lett. B 716 (2012) 30.

[3] Bruno Mansoulié, talk at the Rencontres de Moriond EW 2013, On behalf of the ATLAS collaboration.

[4] [CMS Collaboration], CMS-PAS-HIG-13-005.

[5] [ATLAS Collaboration], ATLAS-CONF-2013-034.

[6] J. Cao et al., JHEP 1203, 086 (2012) [arXiv:1202.5821 [hep-ph]].

[7] U. Ellwanger, JHEP 1203 (2012) 044;

M. Carena et al. JHEP 1203 (2012) 014; 
S. Heinemeyer, O. Stal, G. Weiglein, Phys. Lett. B 710 (2012) 201;

L. J. Hall, D. Pinner, J. T. Ruderman, JHEP 1204 (2012) 131;

A. Arbey, M. Battaglia, F. Mahmoudi, Eur. Phys. J. C 72 (2012) 1906;

A. Arvanitaki, G. Villadoro, JHEP 1202 (2012) 144;

N. D. Christensen, T. Han, S. Su, Phys. Rev. D 85 (2012) 115018;

P. Lodone, Int. J. Mod. Phys. A 27 (2012) 1230010;

K. Hagiwara, J. S. Lee, J. Nakamura, JHEP 1210 (2012) 002;

V. Barger, M. Ishida and W. -Y. Keung, Phys. Rev. D 87 (2013) 015003;

F. Boudjema and G. D. La Rochelle, Phys. Rev. D 86 (2012) 115007;

P. Bechtle et al., Eur. Phys. J. C 73 (2013) 2354;

J. Ke et al., Phys. Lett. B 723 (2013) 113;

K. Cheung, C. -T. Lu and T. -C. Yuan, Phys. Rev. D 87 (2013) 075001;

R. S. Hundi, Phys. Rev. D 87 (2013) 115005;

A. Chakraborty et al., arXiv:1301.2745 [hep-ph];

J. L. Feng, arXiv:1302.6587 [hep-ph];

T. Han, Z. Liu and A. Natarajan, JHEP 1311 (2013) 008;

T. Han, T. Li, S. Su and L. -T. Wang, arXiv:1306.3229 [hep-ph];

K. Kowalska and E. M. Sessolo, arXiv:1307.5790 [hep-ph].

[8] S. F. King, M. Muhlleitner, R. Nevzorov, Nucl. Phys. B 860 (2012) 207;

U. Ellwanger, C. Hugonie, Adv. High Energy Phys. 2012 (2012) 625389;

R. Benbrik et al., Eur. Phys. J. C 72 (2012) 2171;

J. F. Gunion, Y. Jiang, S. Kraml, Phys. Lett. B 710 (2012) 454; JHEP 1210 (2012) 072;

K. Agashe, Y. Cui and R. Franceschini, JHEP 1302 (2013) 031;

T. Gherghetta et al., JHEP 1302 (2013) 032;

S. F. King, M. Mhlleitner, R. Nevzorov and K. Walz, Nucl. Phys. B 870 (2013) 323;

K. Kowalska et al., Phys. Rev. D 87 (2013) 115010;

L. Aparicio et al., JHEP 1302 (2013) 084;

N. D. Christensen, T. Han, Z. Liu and S. Su, JHEP 1308 (2013) 019;

M. Badziak, M. Olechowski and S. Pokorski, JHEP 1306 (2013) 043;

T. Cheng and T. Li, Phys. Rev. D 88 (2013) 015031;

T. Cheng, J. Li, T. Li and Q. -S. Yan, arXiv:1304.3182 [hep-ph]; 
S. Moretti, S. Munir and P. Poulose, arXiv:1305.0166 [hep-ph].

[9] J. Cao et al., JHEP 1304 (2013) 134; JHEP 1211 (2012) 039; arXiv:1301.4641 [hep-ph];

U. Ellwanger, JHEP 1308 (2013) 077; arXiv:1309.1665 [hep-ph];

C. Han et al., arXiv:1304.5724 [hep-ph]; arXiv:1307.3790 [hep-ph]; arXiv:1308.5307 [hep-ph];

W. Wang, J. M. Yang and L. L. You, JHEP 1307 (2013) 158.

[10] J. Cao et al., JHEP 1210 (2012) 079 [arXiv:1207.3698 [hep-ph]].

[11] J. Cao et al., Phys. Lett. B 710, 665 (2012) arXiv:1112.4391 [hep-ph]].

[12] J. Cao, et al., JHEP 1206, 145 (2012); Phys. Rev. D 79, 091701 (2009).

[13] H. Baer et al., Phys. Rev. D 85 (2012) 075010; JHEP 1205 (2012) 091; Phys. Rev. D 87 (2013) 3, 035017;

O. Buchmueller et al., Eur. Phys. J. C 72 (2012) 2020;

S. Akula et al., Phys. Rev. D 85, 075001 (2012);

M. Kadastik et al., JHEP 1205 (2012) 061;

J. L. Feng, K. T. Matchev and D. Sanford, Phys. Rev. D 85 (2012) 075007;

L. Aparicio, D. G. Cerdeno, L. E. Ibanez, JHEP 1204 (2012) 126;

J. Ellis et al., Eur. Phys. J. C 72 (2012) 2005; Eur. Phys. J. C 73 (2013) 2403;

Z. Kang et al., Phys. Rev. D 86 (2012) 095020;

A. Fowlie et al., Phys. Rev. D 86 (2012) 075010;

S. Akula, P. Nath, G. Peim, Phys. Lett. B 717 (2012) 188;

O. Buchmueller et al., Eur. Phys. J. C 72 (2012) 2243.

[14] U. Ellwanger, C. Hugonie and A. M. Teixeira, Phys. Rept. 496, 1 (2010);

M. Maniatis, Int. J. Mod. Phys. A25 (2010) 3505.

[15] J. E. Kim and H. P. Nilles, Phys. Lett. B 138 (1984) 150.

[16] M. Bastero-Gil et al., Phys. Lett. B 489, 359 (2000);

A. Delgado et al., Phys. Rev. Lett. 105, 091802 (2010);

S. F. King and P. L. White, Phys. Rev. D 52 (1995) 4183;

R. Dermisek and J. F. Gunion, Phys. Rev. Lett. 95 (2005) 041801; Phys. Rev. D 76 (2007) 095006;

G. G. Ross and K. Schmidt-Hoberg, Nucl. Phys. B 862 (2012) 710;

Z. Kang, J. Li and T. Li, JHEP 1211 (2012) 024;

A. Farzinnia, H. -J. He and J. Ren, arXiv:1308.0295 [hep-ph]. 
[17] R. Dermisek, J. F. Gunion and B. McElrath, Phys. Rev. D 76 (2007) 051105;

R. Dermisek and J. F. Gunion, Phys. Rev. D 81 (2010) 075003; Phys. Rev. D 79 (2009) 055014

F. Domingo et al., JHEP 0901 (2009) 061;

Z. Heng et al., Phys. Rev. D 77 (2008) 095012;

A. Arhrib et al., JHEP 0703 (2007) 073;

S. Andreas et al., JHEP 1008 (2010) 003;

X. -G. He, J. Tandean and G. Valencia, JHEP 0806 (2008) 002.

[18] F. Mahmoudi, J. Rathsman, O. Stal and L. Zeune, Eur. Phys. J. C 71 (2011) 1608.

[19] D. G. Cerdeno, P. Ghosh and C. B. Park, JHEP 1306 (2013) 031.

[20] D. G. Cerdeno, P. Ghosh, C. B. Park and M. Peiro, arXiv:1307.7601 [hep-ph].

[21] J. -J. Cao et al., Phys. Lett. B 703 (2011) 292 arXiv:1104.1754 [hep-ph]];

J. Kozaczuk and S. Profumo, arXiv:1308.5705 [hep-ph].

[22] M. Almarashi and S. Moretti, Phys. Rev. D 84 (2011) 015014;

C. S. Kim, K. Y. Lee and J. Park, Phys. Rev. D 85 (2012) 117702.

[23] D. A. Vasquez et al., Phys. Rev. D 86 (2012) 035023.

[24] D. J. Miller, R. Nevzorov and P. M. Zerwas, Nucl. Phys. B 681 (2004) 3.

[25] J. Beringer et al. [Particle Data Group Collaboration], Phys. Rev. D 86 (2012) 010001.

[26] U. Ellwanger, J. F. Gunion and C. Hugonie, JHEP 0502, 066 (2005);

U. Ellwanger and C. Hugonie, Comput. Phys. Commun. 175, 290 (2006).

[27] [BaBar Collaboration], Phys. Rev. Lett. 108 (2012) 211801; Phys. Rev. D 88 (2013) 031701;

Phys. Rev. D 87 (2013) 3, 031102; Phys. Rev. Lett. 107 (2013) 221803;

Phys. Rev. D 88 (2013) 071102; Phys. Rev. Lett. 107 (2011) 021804;

Phys. Rev. Lett. 103 (2013) 251801.

[28] [BaBar Collaboration], Phys. Rev. Lett. 109 (2012) 191801;

Phys. Rev. Lett. 109, 101802 (2012).

[29] [LHCb Collaboration], Phys. Rev. Lett. 110 (2013) 021801.

[30] P. A. R. Ade et al. [Planck Collaboration], arXiv:1303.5076 [astro-ph.CO].

[31] E. Aprile, et al. [XENON100 Collaboration], Phys. Rev. Lett. 109 (2012) 181301.

[32] J. Cao et al., JHEP 1007, 044 (2010); Phys. Rev. D 82 (2010) 051701.

[33] P. Bechtle et al., Comput. Phys. Commun. 181, 138 (2010); 
Comput. Phys. Commun. 182, 2605 (2011); arXiv:1305.1933 [hep-ph].

[34] G. Altarelli and R. Barbieri, Phys. Lett. B 253, 161 (1991);

M. E. Peskin, T. Takeuchi, Phys. Rev. D 46, 381 (1992).

[35] LEP and SLD Collaborations, Phys. Rept. 427 (2006) 257.

[36] J. Cao and J. M. Yang, JHEP 0812, 006 (2008).

[37] T. Aaltonen et al. [CDF and D0 Collaborations], arXiv:1303.6346 [hep-ex].

[38] J. Cao et al., JHEP 1308 (2013) 009 arXiv:1303.2426];

X. -F. Han et al., Phys. Rev. D 87 (2013) 055004 [arXiv:1301.0090];

L. Wang, J. M. Yang and J. Zhu, Phys. Rev. D 88 (2013) 075018 [arXiv:1307.7780].

[39] J. R. Espinosa, C. Grojean, M. Muhlleitner, M. Trott, JHEP 1205, 097 (2012);

P. P. Giardino et al., JHEP 1206, 117(2012).

[40] G. Belanger, B. Dumont, U. Ellwanger, J. F. Gunion and S. Kraml, JHEP 1302 (2013) 053.

[41] F. Boudjema et al., arXiv:1307.5865 [hep-ph].

[42] U. Ellwanger, G. Espitalier-Noel and C. Hugonie, JHEP 1109 (2011) 105.

[43] B. Ananthanarayan and P. N. Pandita, Phys. Lett. B 353 (1995) 70;

Phys. Lett. B 371 (1996) 245; Int. J. Mod. Phys. A 12 (1997) 2321.

[44] G. Belanger et al., Phys. Lett. B 723 (2013) 340.

[45] B. Ananthanarayan et al., Phys. Rev. D 87 (2013) 115021.

[46] J. Cao, Z. Heng and J. M. Yang, JHEP 1011 (2010) 110.

[47] K. Cheung, J. Song and Q. -S. Yan, Phys. Rev. Lett. 99, 031801 (2007).

[48] M. Carena, T. Han, G. -Y. Huang and C. E. M. Wagner, JHEP 0804, 092 (2008).

[49] J. Alwall, M. Herquet, F. Maltoni, O. Mattelaer and T. Stelzer, JHEP 1106, 128 (2011).

[50] T. Sjostrand, S. Mrenna and P. Z. Skands, JHEP 0605, 026 (2006).

[51] J. de Favereau et al., arXiv:1307.6346 [hep-ex].

[52] M. Cacciari, G. P. Salam and G. Soyez, Eur. Phys. J. C 72 (2012) 1896.

[53] M. Cacciari and G. P. Salam, Phys. Lett. B 641 (2006) 57.

[54] Similar result is also obtained in, for example, B. Coleppa, F. Kling and S. Su, arXiv:1308.6201 [hep-ph]. 